\title{
Influência do Comonômero e do Método de Polimerização na Cinética de Liberação de Fenobarbitona a partir de Hidrogéis
}

\author{
Ivonete 0. Barcellos \\ Departamento de Química, Universidade Regional de Blumenau (FURB)
}

\author{
Issa A. Katime \\ Grupo de Nuevos Materiales, Faculdad del País Vasco, Espanha \\ Valdir Soldi e Alfredo T. Nunes Pires \\ Grupo de Materiais Poliméricos (Polimat), Departamento de Química, UFSC
}

\begin{abstract}
Resumo: A liberação de drogas solúveis em água a partir de matrizes de hidrogéis hidratados, geralmente envolve simultaneamente a absorção de água e a liberação da droga via mecanismo de difusão controlada. Foi analisado o comportamento difusional da fenobarbitona no hidrogel do copolímero 2-hidroxietil metacrilato (HEMA) e monoitaconato de metila (MMI). A liberação da droga no meio aquoso foi acompanhada por cromatografia líquida de alta pressão e os resultados mostraram que a liberação da fenobarbitona a partir dos hidrogéis estudados, considerando-se os valores dos coeficientes de difusão, não variou com a temperatura e com o método de polimerização. Os valores de energia de ativação sugerem um favorecimento na liberação da fenobarbitona a partir dos hidrogéis de p(HEMA-co-MMI) polimerizados em massa e em solução.
\end{abstract}

Palavras-chave: Liberação controlada, hidrogéis, copolímeros

\section{Influence of Comonomer and Polymerization Method on the Kinetics of Phenobarbitone Released from Hydrogels}

\begin{abstract}
The release of water-soluble drugs from initially hydrated hydrogels matrices generally involves the simultaneous water absorption and drug desorption through a swelling-controlled diffusion mechanism. The diffusional behaviour of phenobarbitone from a hydrogel formed by a 2-hydroxyethylmethacrylate (HEMA) and methyl monoitaconate (MMI) copolymer was reported. The drug release into water was performed by high pressure liquid cromatography and the results showed that the phenobarbitone release from the hydrogels studies, considering the diffusion coefficient values did not change with the temperature and the polymerization method. The activation energy values suggested a more favorable phenobarbitone release from the hydrogels p(HEMA-co-MMI) polymerized in mass and bulk solution.
\end{abstract}

Keywords: Controlled release, hydrogels, copolymers

\section{Introdução}

A utilização de hidrogéis na biomedicina teve como ponto de partida o trabalho pioneiro de Wichterle e Limm $^{[1]}$ na síntese do hidrogel de poli(2-hidroxietil metacrilato) [PHEMA]. A importância dos hidrogéis como biomateriais se deve à semelhança de suas propriedades físicas com os tecidos vivos, tais como elevado conteúdo de água, consistência macia e elástica e baixa tensão interfacial. Em função destas proprieda-

Autor para correspondência: Valdir Soldi, Grupo de Materiais Poliméricos (Polimat), Depto de Química, UFSC, Campus Universitário Trindade, CEP: 88040-900, Florianópolis, SC. E-mail: vsoldi@qmc.ufsc.br 
des, os hidrogéis podem ser aplicados em cateteres, sensores (eletrodos), substratos de culturas de microorganismos, membranas de hemodiálise, lentes de contato flexíveis, enxertos vasculares, imobilização de enzimas e sistemas que liberam drogas, entre ou$\operatorname{tros}^{[2-4]}$. Jeyanthi e Rao ${ }^{[5]}$, prepararam biomateriais a partir da adição de colágeno ao hidrogel PHEMA, sendo os materiais biocompatíveis quando implantados no tecido subcutâneo de camundongos. Hidrogéis de PHEMA e PHEMA-co-AA, onde AA denota a unidade monomérica de ácido acrílico, tem sido estudadas no cultivo de células ${ }^{6}$. Anderson e colaboradore ${ }^{[7]}$ avaliaram o efeito da administração subcutânea de hidrocortisona incorporada ao hidrogel PHEMA na membrana corioalantóide de embriões de frango hipofisectomizados no $18^{\circ}$ dia de gestação, observando um aumento no desenvolvimento muscular desses embriões.

Atualmente um dos grandes interesses na utilização de hidrogéis está relacionado com o processo de liberação controlada de drogas. Em geral, esta aplicação tem como requisitos principais a utilização de materiais que sejam biocompátiveis, apresentem resistência à processos de degradação e adequadas propriedades mecânicas. Dentre os vários mecanismos utilizados na incorporação de drogas à hidrogéis destacam-se: i) o intumescimento do hidrogel em solução contendo a droga e posterior adsorção, e ii) enxerto através de uma reação química. O primeiro mecanismo foi utilizado com sucesso na incorporação da teofilina em hidrogéis de poli (HEMA-co-NVP) ${ }^{[8]}$, (NVP representa N-vinil-2pirrolidona) e do salicilato de sódio no copolímero HEMA/sulfobetaína ${ }^{[9]}$. Estudos de liberação controlada realizados por Giammona e colaboradores ${ }^{[10]}$ através da digestão enzimática de hidrogel PHEMA, utilizando diferentes concentrações de pepsina e alfa-quimotripsina, não mostraram degradação num período de vinte quatro horas e o material formado era biocompatível quando administrado via oral à camundongos.

Glucoamilase (GA), obtidas a partir do Aspergillus niger, foi imobilizada com elevada atividade e estabilidade em microesferas de PHEMA/etileno glicol dimetacrilato (EGDMA) ${ }^{[11]}$. As microesferas de PHEMA ativadas foram intumescidas em solução tampão de fosfato e colocadas em um sistema reator, observando-se que a quantidade e atividade da enzima imobilizada era função do tamanho das microesferas dos hidrogéis. Os autores observaram uma elevada estabilidade operacional do hidrogel no reator com a enzima imobilizada, indicando que PHEMA/EGDMA pode ser utilizado para uma produção contínua de glicose a partir da solução de dextrina.

O objetivo do presente trabalho foi estudar a viabilidade da utilização de hidrogéis formados a partir do poli(2-hidroxietil metacrilato) [PHEMA] e poli(2hidroxietil metacrilato-co-monoitaconato de metila) [p(HEMA-co-MMI)], como sistemas viáveis para incorporação e liberação controlada da droga fenobarbitona. Fenobarbitona é uma droga com ação antiepilética, hipnótico e sedante, com absorção lenta, sendo metabolizada parcialmente no fígado e eliminada principalmente através da urina, numa percentagem da ordem de $25 \%$ na forma inalterada e o restante na forma metabolizada (p-hidroxifenobarbital). Em função das características e aplicações desta droga, a utilização de sistemas otimizados para a incorporação e liberação controlada é de grande importância.

\section{Experimental}

\section{Material e Reagentes}

O monômero 2-hidroxietil metacrilato (HEMA) proveniente da Merck foi previamente purificado conforme descrito por Wichterle ${ }^{[12]}$. O etilenoglicol dimetacrilato (Fluka) e o 2,2' azobisobutironitrilo (AIBN) (Merck) previamente purificado por cristalização em metanol, foram utilizados como agentes reticulante e iniciador da reação de polimerização, respectivamente. $\mathrm{O}$ monoitaconato de metila (MMI) foi sintetizado de acordo com o método descrito por Katime e colaboradores ${ }^{[13]}$.

\section{Metodologia}

O hidrogel PHEMA foi sintetizado a partir do monômero HEMA em presença de EGDMA (1\%), como agente reticulante, através dos métodos de polimerização em massa e em solução. Como iniciador foi utilizado o AIBN, 0,05\% em massa com relação aos monômeros. Na síntese do p(HEMA-co-MMI) foi utilizada a percentagem em massa dos comonômeros HEMA/MMI de 90/10.

Os hidrogéis foram cortados em discos, secos e mantidos em estufa a vácuo até peso constante, na relação espessura/diâmetro de 1/13.

A cinética de intumescimento em água foi acompanhada pela mudança de massa dos discos do hidrogel na presença de diferentes percentagens de EGDMA. 
A fenobarbitona foi incorporada no hidrogel mantendo-se os discos imersos em solução aquosa da droga $(1 \mathrm{mg} / \mathrm{mL})$, durante uma semana. A quantidade de fenobarbitona liberada em função do tempo foi determinada por cromatografia líquida de alta pressão (HPLCPerkin Elmer, bomba série 200 1C, detector UV da Abiappied Biosystems 785A, fase estacionária Sherisorb ODS-C18). A partir de uma curva de calibração, obtida a partir das áreas dos picos dos cromatogramas para concentrações padrões de fenobarbitona na faixa de 1$110 \mu \mathrm{g} / \mathrm{mL}$, foram determinadas as quantidade liberadas da droga em função do tempo. Em todos os cromatogramas monitorados foi observado um único pico correspondente a droga independente da temperatura e do tempo de liberação. Este comportamento sugere também que não ocorreu degradação da droga durante o processo de carga dos hidrogéis, bem como durante os experimentos de liberação.

\section{Resultados e Discussão}

$\mathrm{O}$ aumento de massa dos hidrogéis PHEMA e $\mathrm{p}$ (HEMA-co-MMI) puros e em presença de 0,$5 ; 0,75$; 1,0 e $1,5 \%$ em massa de EGDMA devido ao intumescimento em água, obedeceram uma cinética de segunda ordem, de acordo com a equação 1 ,

$$
\frac{t}{H p}=\frac{1}{k \cdot H p_{\infty}^{2}}+\frac{t}{H p_{\infty}}
$$

onde $H p$ e $H p_{\infty}$ representam as quantidades de água no hidrogel no tempo $t$ e no tempo infinito, respectivamente, e $k$ a constante de velocidade. A partir dos gráficos de $t / H p$ versus $t$ foram determinados os valores da constante de velocidade para o intumescimento do hidrogel em diferentes percentagens de EGDMA e temperaturas. A Figura 1 mostra um gráfico de $k$ em função da percentagem em massa de EGDMA para hidrogéis de PHEMA, em diferentes temperaturas. Embora $k$ seja diretamente proporcional a temperatura, diminui com o aumento da percentagem em massa de EGDMA, devido provavelmente ao aumento do grau de reticulação no sistema. Um comportamento similar foi observado para o copolímero p(HEMA-co-MMI), ou seja, os valores de $k$ são da mesma ordem de grandeza. Com $1 \%$ de EGDMA os valores de $k$ determinados para o copolímero foram iguais a 0,026 e 0,010 a 37 e $22{ }^{\circ} \mathrm{C}$, respectivamente. Estes valores são próximos aos observados para o hidrogel PHEMA (Figura 1), onde $k$ varia de 0,022 a

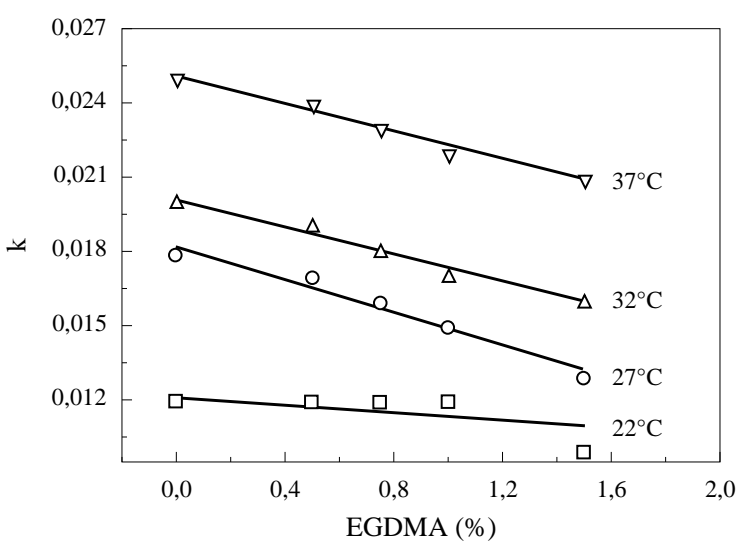

Figura 1. Constantes de velocidade (k) para hidrogéis de PHEMA em função da percentagem em massa de EGDMA a diferentes temperaturas.

0,012, nas mesmas condições. A similaridade observada em termos dos valores de $k$ é condizente com o comportamento observado para a liberação da fenobarbitona conforme discutido a seguir. O mesmo comportamento foi observado para hidrogéis de $\mathrm{N}$-vinil-2-pirrolidona (NVP) e metacrilato de metila em concentrações de reticulante inferiores a $2 \%$. ${ }^{[14]}$

A liberação da fenobarbitona a partir das pastilhas de PHEMA em função de $t^{1 / 2}$ apresentou uma variação linear, considerando-se uma variação unidimensional em função da espessura do disco $(1,1 \pm 0,1 \mathrm{~mm})$. Comportamento similar foi observado no estudo sobre a liberação da teofilina ${ }^{[8]}$, a partir de discos de poli(HEMA-co-NVP) com espessura entre 1-3 mm e na liberação de salicilato sódico a partir de hidrogéis de copolímeros de HEMA com espessura variando de 0,9-2,5 $\mathrm{mm}$.

A Figura 2 mostra a fração de liberação da fenobarbitona em função do tempo, a temperatura constante de $32{ }^{\circ} \mathrm{C}$ e a diferentes percentagens em massa

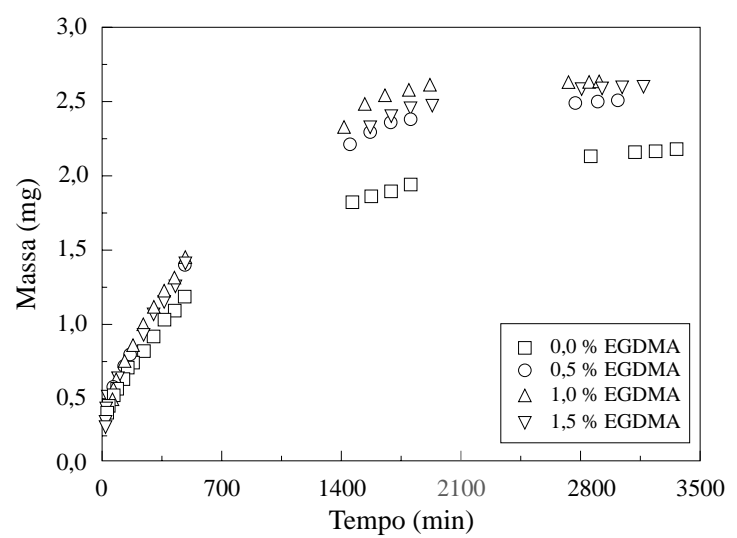

Figura 2. Quantidade de fenobarbitona liberada a partir de hidrogéis de PHEMA em função do tempo para diferentes percentagens em massa de EGDMA, na temperatura de $32{ }^{\circ} \mathrm{C}$. 


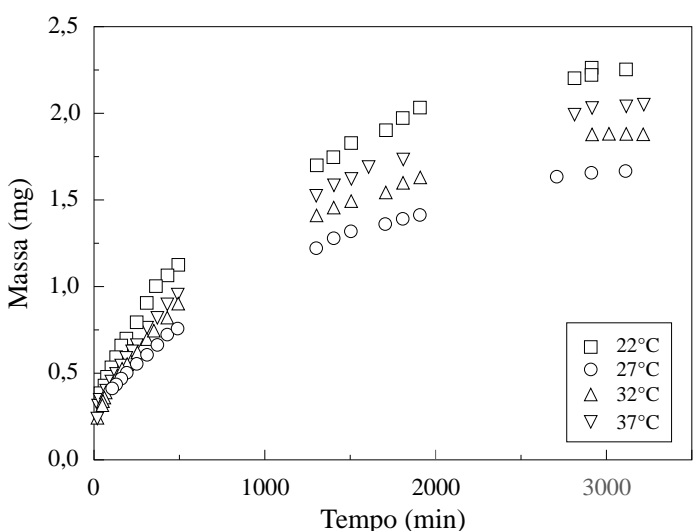

Figura 3. Quantidade de fenobarbitona liberada a partir de hidrogéis de p(HEMA-co-MMI) em função do tempo para diferentes temperaturas e na presença de 1,0 \% de EGDMA.

de EGDMA no hidrogel PHEMA. O comportamento observado indica aparentemente linearidade em função do tempo para valores baixos de liberação. Por outro lado valores constantes independentemente da quantidade de EGDMA presente foi observada após 1800 minutos. O mesmo comportamento foi observado para a liberação da fenobarbitona a partir do hidrogel $\mathrm{p}$ (HEMA-co-MMI) na mesma temperatura e percentagens de EGDMA. A Figura 3 representa a fração de liberação da fenobarbitona em função do tempo, para o hidrogel p(HEMA-co-MMI) contendo 1,0 \% de EGDMA, a diferentes temperaturas. Os coeficientes de difusão aparente para o transporte da fenobarbitona dos hidrogéis para o meio aquoso podem ser determinados a partir da equação 2, onde $F$ é a fração da droga liberada, $M_{t}$ e $M_{\infty}$ são as quantidades (massa) da droga liberada no tempo $t$ e no tempo infinito, respectivamente e $h$ é a espessura da pastilha do hidrogel

$$
F=\frac{M_{t}}{M_{\infty}}=\left(\frac{4 D_{i} t}{\pi h^{2}}\right)^{1 / 2}
$$

carregado com a droga. As Figuras 4 e 5 representam os gráficos de $F$ em função de $t^{1 / 2}$, segundo a equação 2, para os mesmos sistemas e condições descritas nas Figuras 2 e 3 . A partir das inclinações dos gráficos de $F$ em função de $t^{1 / 2}$ foram determinados os valores de $D_{i}$ para os hidrogéis PHEMA e p(HEMA-co-MMI) mostrados na Tabela 1.

Conforme observado para o hidrogel p(HEMAco-MMI, o método de polimerização não afetou os parâmetros cinéticos $\left(D_{i}\right.$ e $\left.E_{a}\right)$ para a liberação da fenobarbitona. Da mesma forma uma variação pouco significativa de $D_{i}$ foi observada devido a presença do comonômero (MMI), ou seja, os coeficientes de
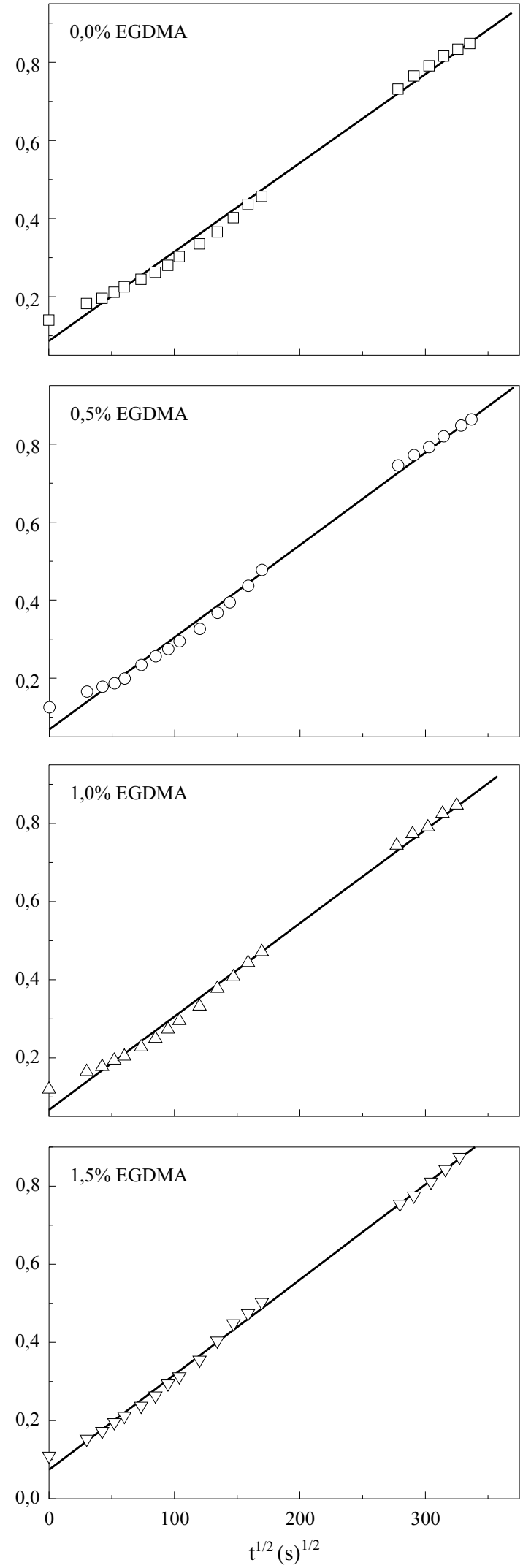

Figura 4. Fração de fenobarbitona liberada em função de $t^{1 / 2}$ para hidrogéis de PHEMA a diferentes percentagens de EGDMA, na temperatura de $32^{\circ} \mathrm{C}$. 

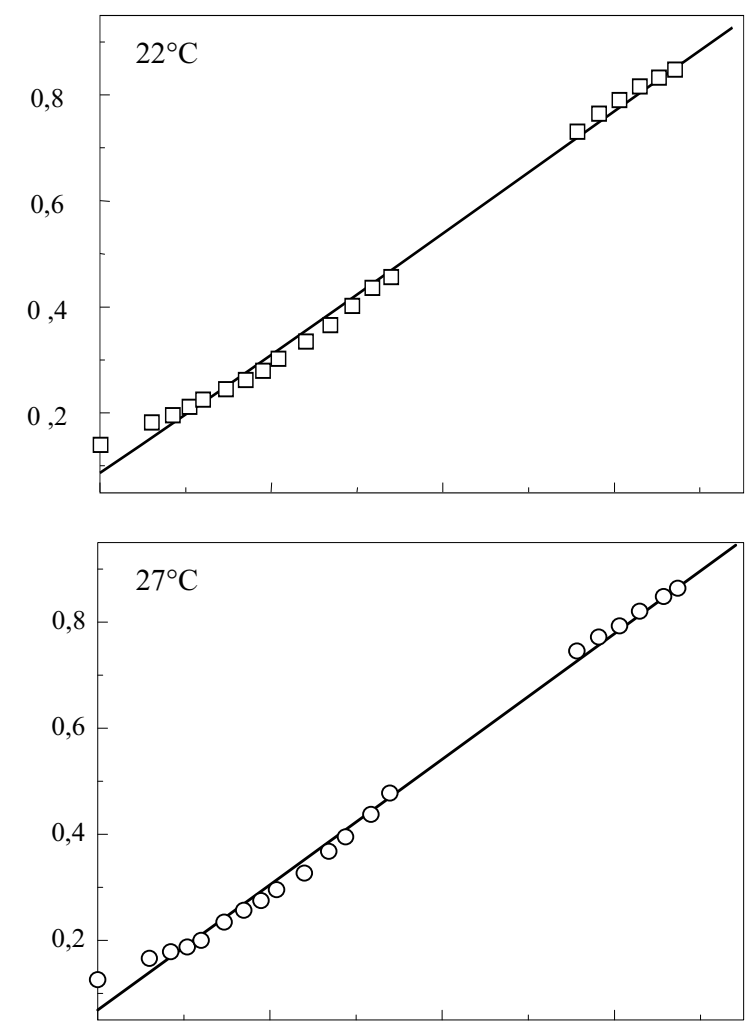

山
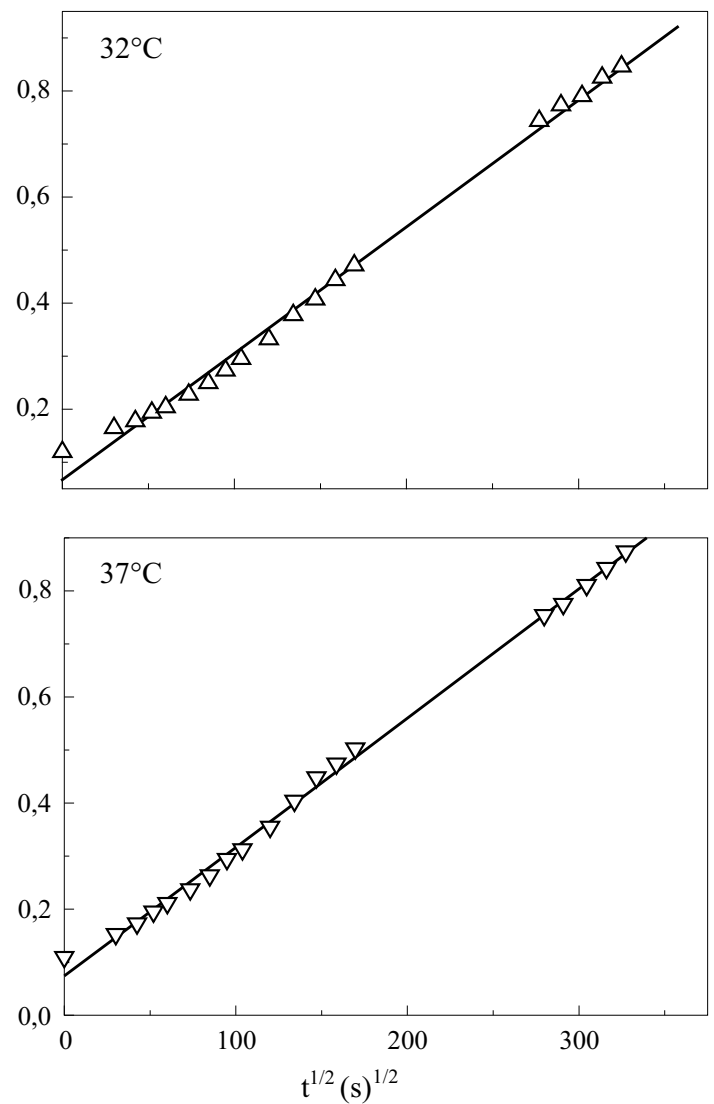

Figura 5. Fração de fenobarbitona liberada em função de $t^{1 / 2}$ para hidrogéis de p(HEMA-co-MMI) polimerizados em massa, a diferentes temperaturas e na presença $1,0 \%$ de EGDMA.
Tabela 1. Parâmetros cinéticos obtidos para liberação da fenobarbitona a partir de diferentes hidrogéis

\begin{tabular}{ccc}
\hline $\begin{array}{c}\text { Hidrogel } \\
\text { PHEMA/MMI }\end{array}$ & $\begin{array}{c}\mathbf{D}_{\mathbf{i}}^{\mathbf{c}} \\
\mathbf{1 0}^{\mathbf{8}}\left(\mathbf{c m}^{\mathbf{2}} \mathbf{~ s}^{-\mathbf{1}}\right)\end{array}$ & $\begin{array}{c}\mathbf{E}_{\mathrm{a}} \\
\left(\mathbf{J ~ m o l}^{-1}\right)\end{array}$ \\
\hline $100 / 0^{\mathrm{a}}$ & 2,0 & 26,2 \\
$90 / 10^{\mathrm{a}}$ & 1,3 & 13,3 \\
$90 / 10^{\mathrm{b}}$ & 1,4 & 12,2 \\
\hline
\end{tabular}

a Polimerização em massa

${ }^{\text {b }}$ Polimerização em solução

${ }^{\mathrm{c}} \mathrm{T}=37^{\circ} \mathrm{C}$

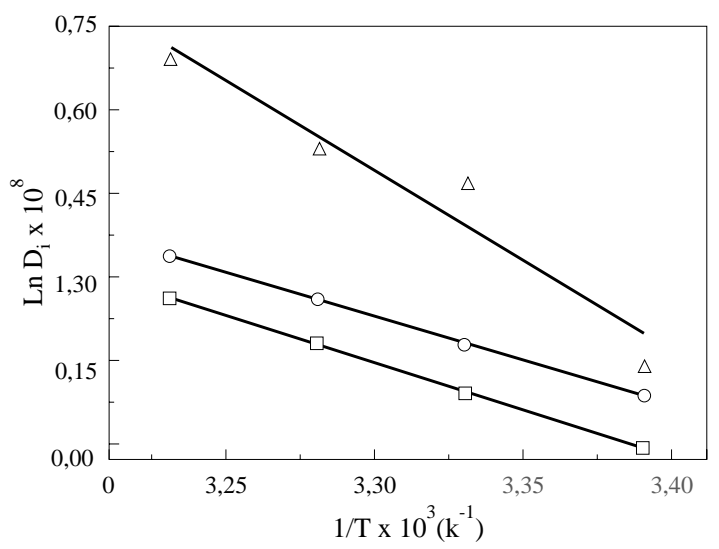

Figura 6. Gráfico do logarítmo natural do coeficiente de difusão em função do inverso da temperatura para diferentes hidrogéis na presença de 1,0 \% em massa de EGDMA: $(\triangle)$ PHEMA polimerizado em solução; (O) p(HEMA-co-MMI) polimerizado em massa e (口) p(HEMA-co-MMI) polimerizado em solução.

difusão para os hidrogéis PHEMA e p(HEMA-coMMI foram próximos, sugerindo uma tendência de favorecimento de liberação devido a presença do comonômero. A energia de ativação $\left(E_{a}\right)$ (Tabela 1$)$ foi determinada considerando- se o comportamento dos coeficientes de difusão em função da temperatura segundo a equação de Arrhenius. Para todos os sistemas estudados os gráficos de $\ln D_{i}$ versus $1 / T$ foram lineares como mostrado na Figura 6 . A redução de $50 \%$ na energia de ativação, sugere que a presença de MMI favorece o processo de difusão da fenobarbitona através do hidrogel, concordando com a tendência observada nos valores de $D_{i}$. De acordo com Graham e McNeil ${ }^{[15]}$ as interações de um hidrogel com uma droga dependem essencialmente da massa molar e da natureza hidrofílica ou hidrofóbica desta. $\mathrm{O}$ favorecimento na liberação da fenobarbitona a partir dos hidrogéis de $\mathrm{p}$ (HEMA-co-MMI) polimerizados em massa ou em solução, em termos da energia de ativação, deve provavelmente estar associado tanto a uma diminuição da afinidade da fenobarbitona por 
este polímero, como pelas possíveis mudanças morfológicas devido a presença do MMI. Por outro lado, outros fatores como a natureza química do hidrogel, do solvente, bem como da droga que será difundida, são fatores determinantes na cinética de liberação controlada.

\section{Conclusões}

A constante de velocidade para o intumescimento dos hidrogéis aumenta com o aumento da temperatura, porém diminui com o aumento da percentagem em massa de EGDMA, devido provavelmente ao aumento do grau de reticulação no sistema. A liberação da fenobarbitona para o meio aquoso a partir dos hidrogéis estudados, considerando-se os valores de $D_{i}$, não variou com a temperatura e com o método de polimerização. Os valores de energia de ativação sugerem um favorecimento na liberação da fenobarbitona a partir dos hidrogéis de p(HEMAco-MMI) polimerizados em massa e em solução. Este comportamento provavelmente está associado tanto a uma diminuição da afinidade da fenobarbitona por este polímero, como pelas possíveis mudanças morfológicas devido a presença do MMI.

A obtenção de hidrogéis que permitam um adequado controle da liberação de drogas como a fenobarbitona pode ser de grande interesse do ponto de vista da otimização dos mesmos para uma posterior utilização em sistemas in vivo. É importante salientar neste caso que os dados obtidos mostraram que esses hidrogéis apresentam uma parcial afinidade pelo fenobarbitona, pois apenas $25 \%$ desta droga foi incorporada aos hidrogéis durante o processo de carga. Embora a liberação tenha sido total e controlável, este tipo de sistema de liberação não seria economicamente o mais viável, pois $75 \%$ da droga está sendo perdida durante o processo de incorporação no hidrogel. Uma alternativa seria utilizar comonômeros que aumentassem significativamente a afinidade pela fenobarbitona ou que morfologicamente fossem mais favoráveis a incorporação desta droga. A continuidade deste trabalho prevê além do estudo da morfologia dos sistemas PHEMA e p(HEMA-co-MMI), a preparação de novos hidrogéis com potencial para otimizar o processo de incorporação e liberação controlada da fenobarbitona.

\section{Agradecimentos}

Os autores agradecem ao CNPq, CAPES e CYTED pelo apoio financeiro.

\section{Referências Bibliográficas}

1. Wichterle, O.; Lim, D. - Nature, 185, 117 (1960).

2. Shenoy, V.; Rosenblatt, J. - Macromolecules, 28, 8751 (1995).

3. Sherwood, J.K.; Zeitlin, L.; Whaley, K.J.; Cone, R. A; Saltzman, M. - Nat. Biotechnol., 14, 468, (1996).

4. Gatenholm, P.; Michalek, J.; Vacik, J. - Macromol. Symp., 109, 127 (1996).

5. Jeyanthi, R.; Rao, K.P. - Biomaterials, 11, 238 (1990).

6. Lombello C.B., Malmonge, S.M., Wade M.L.F. Cytobios, 101, 115 (2000).

7. Anderson, J.M.; Koinis, T.; Nelson, T.; Hosrst, M.; Love, D.S. - ACS Sym. Ser., 31, 167 (1976).

8. Korsmeyer, R.W.; Peppas, N.A. - J. Membrane Sci., 9, 211 (1981).

9. Blanco, M.D., Rego, J.M.; Huglin, M.B. - Polymer, 35, 3487 (1994).

10. Giammona, G.; Pitarresi, G.; Tomarchio, V.; Cacciaguerra, S.; Govoni, P.; J. Pharm. and Pharmacol., 49, 1051 (1997).

11. Arica, M.Y.; Alaeddinoglu, N.G.; Hasirci, V. Enzyme Microb. Technol., 22, 152 (1998).

12. Wichterle, O. - Encyclop. Polym. Sci \& Technol., 15, 273 (1971).

13.Katime, I. Madoz, A.; Velada, J.L. - Thermochim. Acta, 189, 25 (1991).

14. Blanco, M.D.; Trigo, R.M.; Treijon, J.M. - J. Biomaterials Sci. - Polymer Ed., 8, 709 (1997).

15. Graham, N.B.; Mc Neil, M.E. - Biomaterials, 5, 27 (1984).

Recebido: 30/09/99

Aprovado: 06/06/00 